\title{
The Rapid Production of Stress-Free Layers of $\alpha$-Lead Dioxide by Electrodeposition from EDTA-Containing Lead Electrolyte Solutions ${ }^{* 1}$
}

\author{
Minoru UEDA* and Hideo TAKAHASHI
}

Received November 13, 1986 ; Accepted March 5, 1987

\begin{abstract}
This study shows that stress-free layers of $\alpha-1$ ead dioxide could be rapidly produced on a silver-plated base metal by electrodeposition from lead electrolyte solutions containing the sodium salt of ethylenediaminetetracetic acid (EDTA) and that the optimum current density in the constant-potential deposition could be expressed as a linear function of a parameter consisting of the concentrations of lead ion, alkali, and EDTA.
\end{abstract}

\section{INTRODUCTION}

The preparation of a stress-free layer of $\alpha$-lead dioxide $\left(\mathrm{PbO}_{2}\right)$ is an important step in the production of a new type of lead dioxide electrode which has been developed for commercial application by one of the authors (1). The electrode was produced by electrodepositing $\alpha$-lead dioxide and $\beta-1$ ead dioxide successively on a silver-plated base metal. The base chosen was expanded-titanium mesh.

The outstanding property of this electrode was that the contact resistance between adjacent layers of the constituent materials was very small and even the sum total of the resistances was far smaller than the contact resistance found with the electrode produced by electrodepositing $\beta$-lead dioxide on titanium base from the conventional acid bath.

The function of the a-lead dioxide coating -- coating which was produced by electrodeposition from alkaline lead electrolyte solutions -.. was to prevent the silver plate from dissolving during the electrodeposition of $\beta$-lead dioxide from acid lead electrolyte solutions. According to our experience, the $\alpha-1$ ead dioxide deposit found satisfactory for use as the primary coating on the silverplated base metal had no internal stress or a very slight tensile stress.

The electrodeposition of the a-lead dioxide of the required quality is a slow process, insofar as the electrolyte solution described in a previous paper (2) is used. From the viewpoint of time saving, it is desirable to increase the rate of the deposition without impairment of the deposits. Incidentally, Bakhchisaraits yan and Dzhafarov (3) reported that a tensile internal stress was observed in most of the deposits obtained when lead dioxide was electrodeposited from alkaline lead electrolyte solutions containing EDTA. Although not stated definitely in their report, it appears that the deposits consisted chiefly of $\alpha-$ lead dioxide. The highest current density used was $8 \mathrm{~A} \mathrm{dm}^{-2}$, which was far greater than the highest used in our previous work (2). This fact suggests that lead dioxide can be electrodeposited more rapidly from lead electrolyte solutions containing EDTA than from those not containing EDTA. However, the possibility of producing stress-free deposits was not indicated in their report.

This research has, therefore, been carried out in an attempt to find out the conditions for the rapid production of stress-free layers of $\alpha$-lead dioxide by

*1 A paper presented in part at the $51 \mathrm{st}$. National Meeting of the Chemical Society of Japan, Kanazawa, October 1985, Abstr. No. 2B/ 14

National Chemical Laboratory for Industry (Higashi 1-1-4, Yatabe-cho, Tsukuba-gun, Ibaraki-ken 305)

Key Words: $\alpha$-Lead Dioxide, Rapid Electrodeposition, Stress-free 
electrodeposition from alkaline lead electrolyte solutions containing EDTA.

\section{EXPERIMENTAL}

2.1 Determination of Internal Stress.

The internal stress in the lead dioxide deposits was determined by a rapid method (2), which was a modification of the so-called bending strip electrode technique. Thus, lead dioxide was deposited on the bending strip electrode by a constant-potential technique until the amount of electricity passed reached the maximum allowable for obtaining useful data. During the run, the current was periodically interrupted for a short time, and the deflection of the electrode was measured at the moment when the current was stopped. The internal stress was then determined from the deflection measurements.

The electrode was made from a strip of silver $(0.16 \mathrm{~mm}$ in thickness) and its working area was $5 \mathrm{~mm} \times 100 \mathrm{~mm}$. The amount of electricity periodically passed was $50 \mathrm{C}$ and the total amount passed in the whole run was $250 \mathrm{C}$. The amount of an electrolyte solution used for a single run was $1 \mathrm{dm}^{3}$, which was considered to be sufficient enough to minimize the concentration change due to the electrodeposition. The electrodeposition temperature was $60{ }^{\circ} \mathrm{C}$ and the potential range studied was $245 \sim 350 \mathrm{mV}$ vs. SCE. (Both the electrode size and the total amount of electricity were determined by preliminary experiments.)

\subsection{Estimation of the Optimum Potential and the Optimum Current Density.}

The optimum electrode potential for producing stress-free deposits was estimated from a plot of the internal stress against the electrode potential. Similarly, the optimum current density was estimated from a plot of the current density against the electrode potential. The current density used here is the one calculated from the average current strength at the potential in question and the initial working area of the electrode.

\subsection{Electrolyte Solutions.}

The composition of the electrolyte solutions used is shown in Table 1. Solution A was prepared by dissolving lead(II) oxide in the EDTA-containing alkali solution which had been produced by dissolving the disodium salt of EDTA in the proportion of 0.5 mole per liter (M) in $1.5 \mathrm{M}$ sodium hydroxide solution. Solution $B$ was prepared by adding sodium hydroxide to solution $A$. Solution $C$ was made by adding the disodium salt of EDTA to Solution B. Solution $D$ was prepared by dissolving the above-named lead oxide in Solution C. Finally, Solution $E$ was prepared by dissolving the same lead oxide as much as possible in the EDTA-containing alkali solution which had been produced by dissolving the tetrasodium salt of EDTA in the proportion of 0.55 mole per liter in $0.7 \mathrm{M}$ sodium hydroxide solution. The concentrations of sodium hydroxide solutions vere approximate. The lead(II) oxide was litharge for optical use (PbO: $99.5 \%$ at the minimum) and the other chemicals were of reagent grade.

Table 1. The composition of the electrolyte solutions used.

\begin{tabular}{c|ccc}
\hline \multirow{2}{*}{ Soln } & \multicolumn{3}{|c}{ Composition at $60{ }^{\circ} \mathrm{C}$} \\
\cline { 2 - 3 } & $\begin{array}{c}\text { Alka }] \mathrm{i}^{1)} \\
\left(\left[\mathrm{OH}^{-}\right] \tau / \mathrm{M}\right)\end{array}$ & $\begin{array}{c}\text { Lead } \\
\left.\left(\mathrm{Pb}^{2+}\right] \tau / \mathrm{M}\right)\end{array}$ & $\begin{array}{c}\text { EDTA } \\
{\left[\left(\mathrm{EDTA}_{\mathrm{T}} / \mathrm{M}\right)\right.}\end{array}$ \\
\hline A & 1.18 & 0.44 & 0.50 \\
B & 1.68 & 0.44 & 0.50 \\
C & 1.40 & 0.44 & 0.73 \\
D & 1.60 & 0.66 & 0.73 \\
E & 1.85 & 0.54 & 0.55 \\
\hline
\end{tabular}

1) Determined by titration with nitric acid solution.

2) Determined by the procedure described in Ref. (4).

\section{RESULTS AND DISCUSSION}

The lead dioxide deposit produced on the bending strip electrode in every run of the deflection measurement was found to consist of the $\alpha$-modification by the powder $x$-ray diffraction method. Since the crystalline modification was the 
same as that of the dioxide electrodeposited from the alkaline electrolyte solutions without any addition of EDTA (5), it was considered that the organic complexing agent had no effect of changing the crystalline modification.

The internal stress varied from tensile to compressive as the anode potential or the current density was increased. The trend of the variation was the same as that observed in the electrodeposition from the solutions containing no EDTA. An example of this trend is shown in Fig. 1. The symbols $E_{0}$ and $j_{0}$ in the figure represent, respectively, the optimum anode potential and the corresponding current density for producing the stress-free deposits. Figure 1 also shows the method of obtaining the values of $E_{0}$ and $j_{0}$.

The values of $E_{0}$ and $j_{0}$ thus obtained for the electrodeposition from different solu-

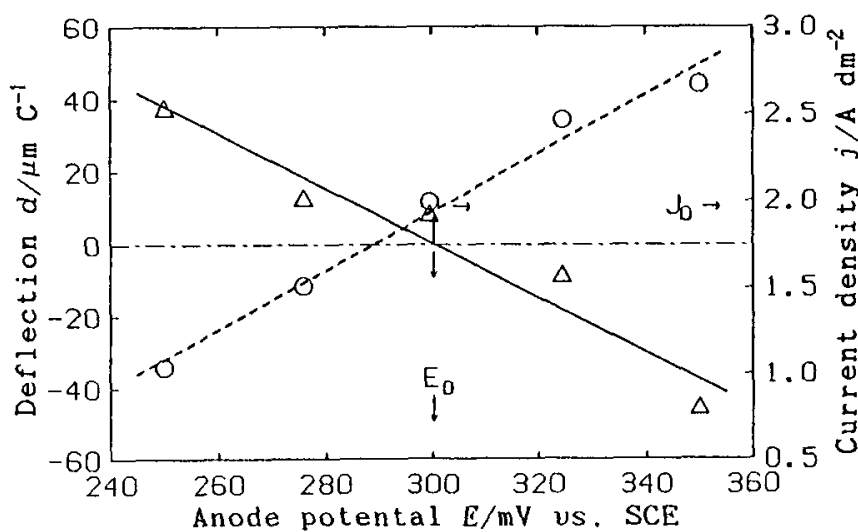

Fig. 1 Variations of deflection and current density with anode potential (Soln A).

$$
\Delta \text { : Deflection, } 0 \text { : current density. }
$$
tions are given in Table 2

The composition of the solutions is also shown in the table, since our primary interest is in the relationship between $j_{0}$ and the composition.

Table 2. The optimum potential and the optimum current density for producing the stress-free $\alpha$-lead dioxide layers.

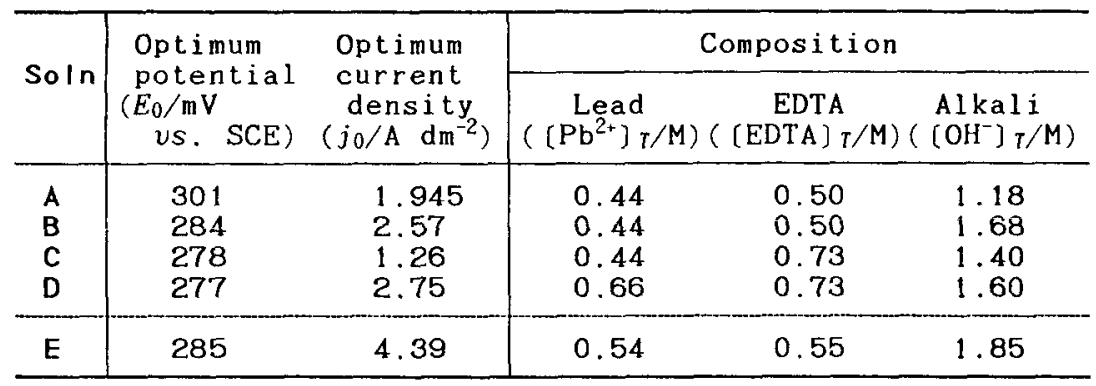

The data in Table 2 are not numerous enough to permit us to drav a clear inference about the relationship between the optimum current density and the electrolyte composition. However, it was suspected that there might be a simple correlation between $j_{0}$ and the composition.

In the following an attempt is made to derive such a correlation under certain simplifying assumptions.

The assumptions required are as follows:

(a) The majority of lead ions in the electrolyte solution exists in the form of 1:1 lead--EDTA complex and a greater part of the remainder in the form of $1: 1$ lead-hydroxo complex. However, as a rough approximation, the concentration of the EDTA complex may be regard as being nearly equal to the total lead ion concentration.

(b) The lead--hydroxo complex yields lead dioxide upon anodic oxidation, but the EDTA complex will not readily yield the dioxide, under the electrolysis con-- 
ditions used.

(c) For convenience' sake, hydrogenplumbite ion (which may readily be formed by the interaction of $1: 1$ lead--hydroxo complex with excess $\mathrm{OH}^{-}$) can be treated as $1: 1$ lead--hydroxo complex.

(d) Over the anode potential range studied, $j_{0}$ is roughly proportional to the bulk concentration of the hydroxo complex.

Here, let $M, L E$, and $L_{H}$ denote $\mathrm{Pb}^{2+}$, EDTA (a ligand), and $\mathrm{OH}^{-}$(a ligand), respectively; and also let the subscript $T$ used with the concentration symbol (square brackets) denote the total concentration. (As is usual, the charges of ions are omitted for the sake of simplicity.)

Then, under the assumptions (a) and (c), the complexation equilibria can be represented by

$$
\begin{aligned}
& \frac{\left[M L_{E}\right]}{[M]\left[L_{E}\right]}=K_{E} \\
& \frac{\left(M L_{H}\right]}{[M]\left[L_{H}\right]}=K_{H}
\end{aligned}
$$

where $K_{E}$ and $K_{H}$ are the stability constants of the complexes. In this case, the concentrations of all the chemical species are also governed by the following conditions:

$$
\begin{aligned}
& {\left[L_{E}\right]=\left[L_{E}\right]_{T}-\left[M L_{E}\right]} \\
& {\left[L_{H}\right]=\left[L_{H}\right]_{T}-\left[M L_{H}\right]} \\
& {[M]_{T}=\left[M L_{E}\right]}
\end{aligned}
$$

Eliminating (M) from Equations (1) and (2), and substituting Equations (3) and (4) in the resulting equation, one obtains after some reductions Equation (6) for the molar ratio of the two complexes.

$$
\frac{\left[M L_{H}\right]}{\left[M L_{E}\right]}=\frac{K}{R} \frac{\left[L_{H}\right] T}{\left(L_{E}\right] T}
$$

where $K=K_{H} / K_{E}$ and $R=1+(K-1)\left[M L_{E}\right] /\left[L_{E}\right]_{T}$.

$$
\begin{aligned}
& \text { The substitution of Equation (5) in Equation (6) gives } \\
& \qquad \frac{\left[M L_{H}\right]}{[M] T} \fallingdotseq \frac{K\left(L_{H}\right] T}{R}\left[L_{E}\right] T
\end{aligned}
$$

and accordingly,

$$
j_{0} \propto \frac{\mathrm{K}}{\mathrm{R}} \frac{[\mathrm{LH}] T[\mathrm{M}) T}{[\mathrm{LE}] T}
$$

or

$$
j_{0} \propto \frac{\mathrm{K}}{\mathrm{R}} \frac{\left(\mathrm{OH}^{-}\right] T\left(\mathrm{~Pb}^{2+}\right] \tau}{[\mathrm{EDTA}] \tau}
$$

From Equation (8), it is expected that $j_{0}$ will vary linearly with the parameter $\left[L_{H}\right]_{T}(M]_{T} /\left[L_{E}\right]_{T}$, insofar as the term $\mathrm{R}$ remains nearly constant. Actually, in the case of Solutions A, B, C, and D, jo varied almost linearly with this parameter (Fig. 2). The linear relationship was considered very satisfactory, because the regression coefficient was as great as 0.975 . However, when Equation (8) was tested for its validity with another solution (E), it was found that the observed value of $j_{0}$ was considerably greater than predicted by the extrapolation of the best fit line drawn through the previous four points, and the deviation was $28 \%$ of the predicted value. On the other hand, when the best 
f:t line was drawn through all the five points shown, the greatest difference between the observed and estimated values of $j_{0}$ was $15 \%$ of the estimated value. Again in this case, the linear relationship was considered satisfactory, because the regression coefficient was 0.960 . Therefore, it is considered that the linear relationship will hold unless the composition is varied extremely.

Equation (8) indicates that jo can be increased by increasing either $\left[L_{H}\right]_{T}$ or the ratio $[M] T /\left[L_{E}\right] \tau$, or by increasing both. However, because of the low solubility of divalent lead in alkaline solution, [M] $T$ alone cannot be increased beyond a certain limit. In order for $[M]_{T}$ to be increased, [LE] $r$ must be increased. Therefore, it will be impossible to increase the ratio $(M]_{T /}\left(L_{E}\right]_{T}$ beyond a certain limit. As shown in Fig. 2, the highest $j_{0}\left(4.4 \quad \mathrm{~A} \mathrm{dm}^{-2}\right)$ was observed when Solution $E$ vas used. Despite the relatively low total alkaline concentration (1.85 M) of the electrolyte solution, it was 1.6 times as large as $j_{0}$ observed in our previous experiment (2) with an alkaline saturated solution of lead(II) oxide containing no EDTA $\left(\left[\mathrm{OH}^{-}\right]_{T}=3.5 \mathrm{M},\left(\mathrm{Pb}^{2+}\right)_{T}=0.22 \mathrm{M}\right)$.

In conclusion, the addition of EDTA to the lead electrolyte solutions was found to facilitate the rapid production of stress-free deposits of $\alpha$-lead dioxide and, at the same time, the sparing use of alkali. The optimum current density for producing the stress-free deposits was expressed as a linear function of the parameter, $\left[\mathrm{OH}^{-}\right] \tau\left[\mathrm{Pb}^{2+}\right] \tau /[\mathrm{EDT}] \tau$. Moreover, although not mentioned earlier in this paper, EDTA was found to be quite stable under the conditions of electrolysis.

\section{REFERENCES}

1) A. Fukasawa, M. Ueda and A. Watanabe, Nippon Kogyo Kaishi, 100, 599 (1984).

2) M. Ueda, A. Watanabe and A. Fukasawa, Denki Kagaku, 52, 779 (1984).

3) N. G. Bakhchisaraits yan and E. A. Dzhafarov, Dokl. Akad. Nauk Azerb. SSR., $18(6), 27$ (1962); Chem. Abstr., 58, 225c (1963).

4) J. Kinnunen and B. Merikanto, Chemist Analyst, 44(3) 75 (1955).

5) A. Fukasawa, Jpn. Tokkyo Koho 80-9,472; USP 4,064,035 (1977). 